\title{
Redução de Ruído em Implantes Cocleares utilizando uma Rede Neural Recorrente baseada na Estimação da Envoltória da Fala
}

\author{
Paulo Henrique Gubert e Márcio Holsbach Costa
}

\begin{abstract}
Resumo - Este trabalho apresenta um método de redução de ruído para implantes cocleares baseado em uma rede neural recorrente. $O$ aprendizado é realizado a partir de estimativas da potência da fala contaminada e de uma máscara tempo-frequência ideal, que tem como objetivo a estimação da envoltória da fala. Simulações computacionais usando um critério objetivo indicam aumento significativo de inteligibilidade em comparação à implementação prática (usando um estimador real da razão sinal ruído) da máscara utilizada no treinamento. Os resultados obtidos sugerem que o desempenho da rede neural recorrente se aproxima ao da máscara ideal nas condições analisadas.
\end{abstract}

Palavras-Chave - Implante coclear, redução de ruído, redes neurais.

Abstract - This work presents a noise reduction method for cochlear implants using a recurrent neural network. Learning is performed from estimates of the noisy speech power and an ideal time-frequency mask, which aims to estimate the speech envelope. Computational simulations using an objective criterion indicate a significant intelligibility increase in comparison with the practical implementation (using a real signal to noise ratio estimator) of the mask employed in the training phase. The results obtained suggest that the recurrent neural network performance gets closer of the ideal mask in the analyzed scenarios.

Keywords - Cochlear implant, noise reduction, neural networks.

\section{INTRODUÇÃO}

Implantes cocleares são próteses eletrônicas cirurgicamente implantáveis que têm como objetivo a restauração da audição em pacientes com perdas profundas ou severas [1] [2].

A diferença entre um aparelho auditivo e um implante coclear (IC) consiste no tipo de estímulo aplicado ao usuário. Enquanto no primeiro o estímulo é feito por ondas sonoras enviadas ao canal auditivo, no segundo o nervo auditivo é diretamente estimulado por corrente elétrica aplicada através de eletrodos posicionados no interior da cóclea.

Em situações ideais de conversação, ou seja, sem a presença de ruído, os usuários de IC reconhecem cerca de $80 \%$ das palavras, o que permite a conversação normal. Entretanto, a existência de ruído de fundo pode afetar significativamente a inteligibilidade, reduzindo o reconhecimento de palavras para cerca de $20 \%$ em situações de razão sinal ruído (SNR - signal to noise ratio) de $5 \mathrm{~dB}$ [3]. Isso ocorre em função da reduzida resolução espectral e temporal do sinal recebido pelo usuário, devido à limitação do número de canais implantados, que pode variar de 16 a 64 [1]. Adicionalmente, o limiar de recepção da

Paulo Henrique Gubert e Márcio Holsbach Costa, Programa de PósGraduação em Engenharia Elétrica, Universidade Federal de Santa Catarina, Florianópolis-SC, e-mails: p.h.gubert@posgrad.ufsc.br, costa@eel.ufsc.br. Este trabalho foi parcialmente financiado pela CAPES e CNPq (315020/2018-0). fala (SRT - speech reception threshold), que indica a SNR na qual $50 \%$ da fala é reconhecida, é cerca de 7 a $20 \mathrm{~dB}$ maior do que em ouvintes normais [4]. A partir do exposto, há a necessidade de incorporação de sistemas de redução de ruído em ICs de forma a permitir a necessária comunicação em ambientes ruidosos.

Máscaras tempo-frequência constituem uma importante classe de métodos de redução de ruído, com ampla aplicação em sistemas de auxílio à audição [5]. Nessa abordagem, o sinal é decomposto em unidades de tempo e frequência e um fator de atenuação (geralmente entre 0 e 1), calculado a partir da SNR local, é aplicado. As máscaras mais usuais são o filtro de Wiener (WF - Wiener filter) e suas variantes [6] e a máscara binária [7]. Porém, recentemente, foi demonstrado que estratégias baseadas na envoltória da fala resultam em uma maior inteligibilidade em indivíduos implantados [8].

A máscara $\mathrm{C} 2 \mathrm{~F}$ [8] é obtida a partir da minimização do erro quadrático médio entre o quadrado da envoltória temporal ideal e da estimada do sinal de fala, em cada canal de estimulação, resultando em aumento significativo de inteligibilidade em situações de baixa SNR em comparação ao WF. Apesar de certa robustez em relação a erros de estimação da SNR [9], seu desempenho ainda assim é afetado negativamente e, portanto, depende diretamente do estimador de SNR utilizado.

Com os avanços recentes na miniaturização e aumento de poder computacional dos novos microprocessadores dedicados a aplicações de implantes cocleares, métodos de redução de ruído baseados em redes neurais começam a ser propostos [10] [11]. As estratégias mais utilizadas são: (a) processamento direto da fala, no qual a rede atua sobre o sinal ruidoso apresentando em sua saída a fala estimada [12]; (b) estimação de máscara tempo-frequência, na qual a rede estima uma determinada máscara, diretamente a partir do sinal ruidoso (sem uso de estimador de SNR) [13]. Esta última abordagem resulta em redes de menor complexidade, mais adequadas à implementação em ICs.

Estudos recentes têm apontado a conveniência de redes neurais recorrentes mimetizando a máscara de Wiener em aplicações de ICs. Apesar das várias contribuições apresentadas na literatura, até o momento não foram realizados estudos relativos à máscara $\mathrm{C} 2 \mathrm{~F}$, mais apropriada à aplicação em questão.

Desta forma, neste trabalho é apresentada uma rede neural recorrente para a estimação da máscara $\mathrm{C} 2 \mathrm{~F}$ (baseada na envoltória da fala). Na Seção II é descrita a estrutura de um sistema de redução de ruído, para implantes cocleares, baseado em máscara tempo-frequência; enquanto que na Seção III é apresentada a proposta baseada em uma rede neural recorrente. $\mathrm{Na}$ Seção IV são descritas as simulações computacionais 
utilizadas para a verificação de desempenho e na seção V são apresentados os resultados obtidos. Por fim, a Seção VI apresenta a conclusão do trabalho.

Ao longo desse texto, letras minúsculas em itálico são variáveis escalares; maiúsculas em itálico são constantes; letras maiúsculas e minúsculas em negrito representam, respectivamente, matrizes e vetores.

\section{MÁscaras TEMPO-FrequÊNCIA}

A fala contaminada por ruído aditivo é representada na forma $y(n)=x(n)+v(n)$, em que $x(n)$ é a fala de interesse, $v(n)$ é o ruído e $n$ é o tempo discreto. Assume-se que ambos os sinais $x(n)$ e $v(n)$ são não-observáveis individualmente e sem correlação entre si. O sinal $y(n)$ é processado por um banco de $k=1, \ldots, K$ (em que $K$ é o número de canais) filtros passa-faixa, apresentando na saída de cada canal o sinal $y_{k}(n)=x_{k}(n)+v_{k}(n)$ limitado em banda.

O sinal de fala em cada canal é estimado através de um processo de filtragem definido por:

$$
\hat{x}_{k}(n)=c_{k}(n) y_{k}(n),
$$

em que $c_{k}(n)$ é o coeficiente único do filtro de estimação e $\hat{x}_{k}(n)$ é a estimativa do sinal de interesse de $x_{k}(n)$.

O coeficiente $c_{k}(n)$ pode ser interpretado como uma máscara tempo-frequência, com valores no intervalo [0,1], que tem como função atenuar canais com baixa $\operatorname{SNR}\left(c_{k}(n)<1\right)$ e preservar canais com alta $\operatorname{SNR}\left(c_{k}(n) \cong 1\right)$.

A envoltória de cada um desses sinais é dada por [14]:

$$
\hat{x}_{\mathrm{e} k}(n)=\left|\hat{x}_{\mathrm{a} k}(n)\right|=\sqrt{\hat{x}_{k}^{2}(n)+\left[H\left\{\hat{x}_{k}(n)\right\}\right]^{2}},
$$

em que $|\cdot|$ representa o módulo; $H\{\cdot\}$ é o operador da transformada de Hilbert; $\hat{x}_{\mathrm{a} k}(n)$ é o sinal analítico de $\hat{x}(n)$. As envoltórias temporais $\hat{x}_{\mathrm{e} k}(n)$ são utilizadas para a modulação dos trens de pulsos que acionam as fontes de corrente para estimulação da cóclea.

\section{A. Máscara $C 2 F$}

Os coeficientes do filtro de redução de ruído $c_{k}(n)$ podem ser calculados de diferentes maneiras, de acordo com critérios de projeto. A máscara $\mathrm{C} 2 \mathrm{~F}$ minimiza o erro quadrático médio entre os quadrados das envoltórias temporais do sinal estimado e da fala de interesse, apresentando a seguinte função custo [8]:

$$
J=E\left\{\left|x_{a k}(n)\right|^{2}-\left|\hat{x}_{a k}(n)\right|^{2}\right\},
$$

em que $E\{\cdot\}$ é o valor esperado. A minimização de (3) resulta em:

$$
c_{k}(n)=\sqrt{\frac{2 \xi_{k}^{2}(n)+\xi_{k}(n)}{2 \xi_{k}^{2}(n)+4 \xi_{k}(n)+2}},
$$

em que $\xi_{k}(n)=\sigma_{\mathrm{x} k}^{2}(n) / \sigma_{\mathrm{v} k}^{2}(n)$ é a SNR a priori local (no canal índice $k) ; \quad \sigma_{\mathrm{x} k}^{2}(n)=E\left\{x_{k}^{2}(n)\right\} \quad$ e $\quad \sigma_{\mathrm{v} k}^{2}(n)=E\left\{v_{k}^{2}(n)\right\}$ são, respectivamente, as variâncias da fala e do ruído em cada um dos $k$ canais.

A estimativa da SNR a priori é comumente realizada pelo método da decisão direta [15]:

$$
\hat{\xi}_{k}(n)=a \hat{\xi}_{k}(n-1)+(1-a) \max \left\{\left[\hat{\gamma}_{k}(n)-1,0\right], \xi_{\min }\right\},
$$

em que $a$ é um parâmetro de suavização; $\xi_{k}(n)=\hat{\sigma_{\mathrm{x}}}{ }^{2}(n) / \hat{\sigma}_{\mathrm{v}} k^{2}(n)$ é uma estimativa da SNR a priori local no instante $n$; $\hat{\gamma_{k}}(n)=\hat{\sigma}_{\mathrm{y} k}^{2}(n) / \hat{\sigma}_{k}^{2}(n)$ é uma estimativa da SNR a posteriori local no instante $n ; \max \{\cdot, \cdot\}$ é o maior número entre os dois argumentos da função; $\xi_{\min }$ é a SNR a posteriori mínima.

\section{Sistema PRoposto}

A Fig. 1 apresenta a estrutura do sistema de redução de ruído proposto para implantes cocleares. São destacados quatro blocos de processamento. $\mathrm{O}$ caminho direto de processamento da fala contaminada é definido pela seguinte sequência: (a) redução de ruído: composto por um banco de filtros utilizado para a decomposição do sinal em múltiplas bandas e a aplicação da máscara tempo-frequência definida pela saída da rede neural recorrente; (b) estimação de envoltória: baseada no módulo do sinal analítico, calculado utilizando a transformada de Hilbert; (c) estimulação da cóclea: que aplica uma estratégia de estimulação (e.g. continuous interleaved sampling ou advanced combination encoders [2]) e conversão para a forma de trem de impulsos de corrente.

O bloco denominado de "processo de aprendizagem" é utilizado previamente ao funcionamento do sistema, proporcionando treinamento para a rede neural. Para o cálculo da SNR ideal é utilizado o conhecimento individualizado dos sinais de fala e ruído em cada banda. Esse valor é utilizado em (4) para obter a máscara C2F ideal [8]. Após a finalização do treinamento, este bloco é retirado do sistema.

A rede neural utilizada é do tipo recorrente, utilizada de forma bem sucedida em conjunto com a máscara de Wiener ideal em [13].

\section{SIMULAÇÕES COMPUTACIONAIS}

Esta seção apresenta a configuração dos experimentos computacionais utilizados para a avaliação da estrutura proposta. Comparações quanto à inteligibilidade são realizadas entre: (a) a fala original; (b) a fala contaminada; a (c) fala contaminada processada pelo método proposto; (d) a fala contaminada processada pelo método $\mathrm{C} 2 \mathrm{~F}$ utilizando um estimador de SNR ideal; (e) a fala contaminada processada pelo método $\mathrm{C} 2 \mathrm{~F}$ utilizando um estimador de SNR real.

O banco de filtros utilizado é do tipo Gammatone com $K=22$ canais, utilizando escala ERB (em inglês, Equivalent Rectangular Bandwidth) para simular o sistema auditivo humano [16].

\section{A. Banco de dados}

O conjunto de sinais utilizado é o IEEE corpus que possui 720 arquivos de fala [17]. Foi utilizado ruído de balbuciação para contaminação da fala, obtido em uma cafetaria com a presença de múltiplos locutores [17]. Os sinais foram reamostrados para $16 \mathrm{kHz}$ e contaminados para SNR $\in\{-6$, $-3,0,3,9\} \mathrm{dB}$, de forma a realizar o processo de treinamento e para $\mathrm{SNR} \in\{-3,3,9\} \mathrm{dB}$ com a finalidade de teste [18].

Foram criados 720 arquivos de sinais contaminados para cada SNR, sendo que $70 \%$ deles foram utilizados para treinamento e $30 \%$ para teste, totalizando assim, um banco de dados com 3168 sinais. 


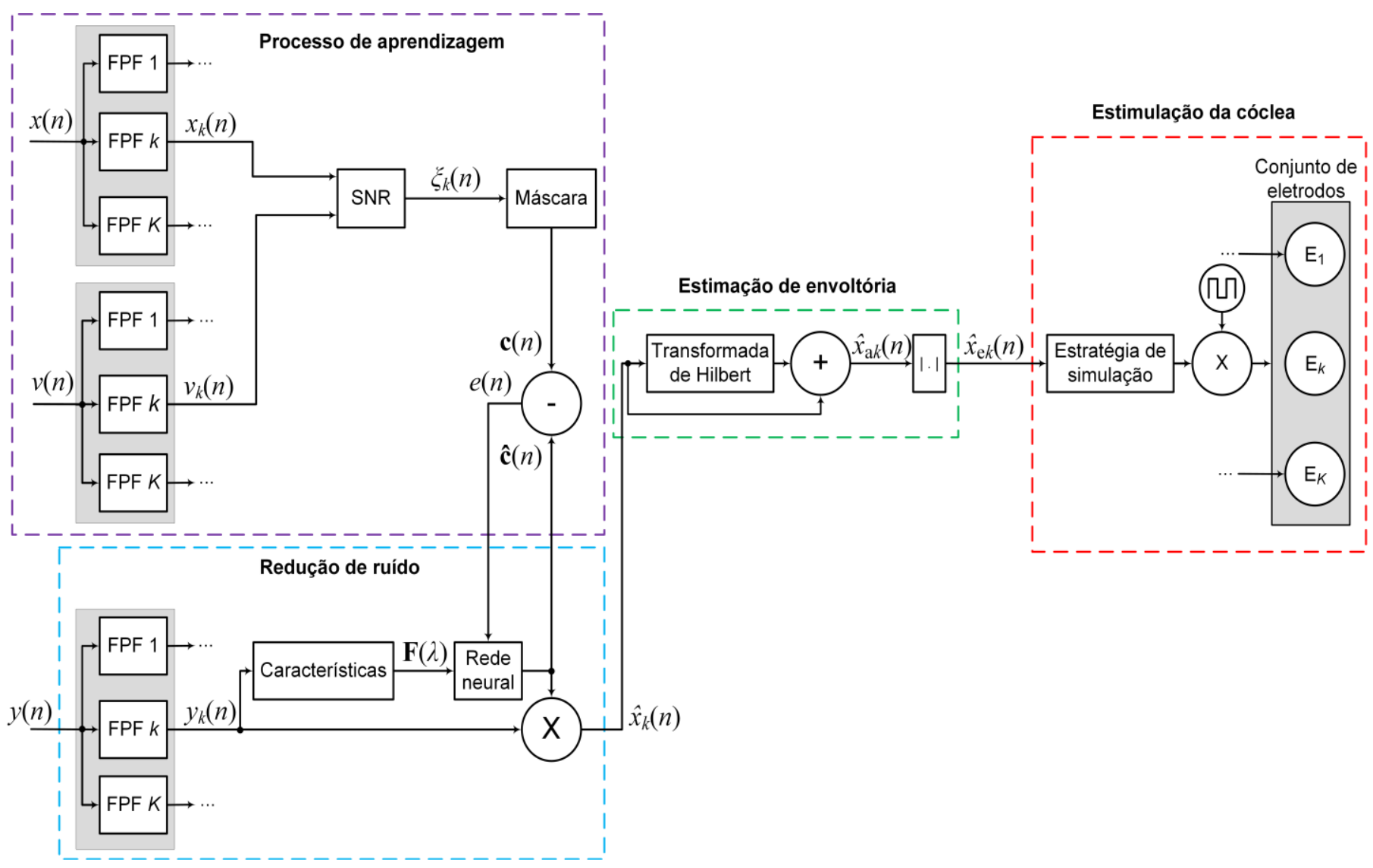

Fig. 1: Estrutura de processamento baseada em [8].

\section{B. Rede neural}

A rede neural utilizada é do tipo Long Term Short Memory (LTSM), possuindo memória de curta e longa duração [8]. Foram utilizados os seguintes parâmetros: camada de entrada do tipo sequencial; 2 camadas ocultas do tipo LTSM com 128 unidades cada; 1 camada de saída totalmente conectada (densa) de dimensão 22. Além disso, foram adicionadas duas camadas de dropout com fator de esquecimento de $20 \%$, sendo esse um valor típico para processamento de fala [19].

Os dados de predição foram normalizados para terem média nula e variância unitária. O número de épocas foi de 20 e o minibatch possui tamanho 8. A função custo a ser minimizada foi o erro quadrático médio. A rede foi desenvolvida utilizando a biblioteca TensorFlow e o processamento dos dados foi feito no Google Colab.

Como vetor de características [20] foi utilizada a informação da energia estimada nos $K$ canais (em um trecho de $N=320$ amostras) no instante atual e em $Q=4$ quadros passados, obtidos a partir de trechos sem sobreposição. A partir dessas considerações, a matriz de informação para treinamento da rede foi definida como:

$$
\mathbf{F}(\lambda)=\left[\begin{array}{llll}
\mathbf{f}(\lambda) \mathbf{f}(\lambda-1) & \ldots & \mathbf{f}(\lambda-Q)
\end{array}\right],
$$

em que:

$$
\mathbf{f}(\lambda)=\left[\begin{array}{lll}
\sum_{i=(\lambda-1) N+1}^{\lambda N} y_{1}^{2}(i) & \ldots & \sum_{i=(\lambda-1) N+1}^{\lambda N} y_{K}^{2}(i)
\end{array}\right]^{\mathrm{T}} .
$$

O treinamento da rede é realizado a partir do conhecimento individualizado da fala e do ruído de contaminação, utilizando a SNR ideal para calcular a máscara C2F. Os sinais de erro entre o coeficiente ótimo da máscara $\mathrm{C} 2 \mathrm{~F}$ e a saída da rede $e_{k}(n)$ são utilizados para o processo de aprendizado. Após a finalização do processo de treinamento esse bloco é desabilitado.

É utilizada a técnica de parada antecipada, ou seja, se o erro de validação aumentar por 3 épocas seguidas, o treinamento é interrompido.

\section{Máscara $C 2 F$}

A máscara $\mathrm{C} 2 \mathrm{~F}$ é implementada de forma ideal, da mesma forma descrita para o treinamento da rede; como também através do método de decisão direta, conforme descrito em (5). Foram utilizados os seguintes parâmetros: $\xi_{\min }=0,0318$ $(-15 \mathrm{~dB})$ e $a=0,98$ [21]. A densidade espectral de potência do ruído é estimada usando o método de estatísticas regionais [21] [22].

\section{Critério objetivo de inteligibilidade}

Para estimação da inteligibilidade foi utilizado o critério SRMR-CI por apresentar alta correlação com a inteligibilidade de usuários de IC [23]. A SRMR-CI pode ser mapeada em valores de inteligibilidade por:

$$
I(\%)=\frac{100}{1+e^{\left(a_{1} S R M R-C I_{\text {norm }}+a_{2}\right)}},
$$

em que $I$ é o valor de inteligibilidade porcentual; $a_{1}$ e $a_{2}$ são parâmetros de ajuste, definidos por um modelo de regressão linear entre as pontuações das medidas objetivas e subjetivas; por fim, o resultado é normalizado para o intervalo entre $[0,1]$. 


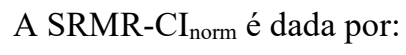

$$
S R M R-C I_{\text {norm }}=\frac{S R M R-C I_{\mathrm{t}}}{S R M R-C I_{\mathrm{c}}},
$$

em que $S R M R-C I_{\mathrm{t}}$ é a métrica obtida para o sinal sob teste e $S R M R-C I_{\mathrm{c}}$ é para a fala não contaminada [23].

\section{E. Análise estatística}

Foi realizada a comparação de múltiplos espaços amostrais usando a abordagem de amostras dependentes, pois os mesmos sinais são aplicados em todos os métodos de análise. O procedimento é dividido em duas etapas: detecção de diferença estatística entre os grupos e teste de comparações múltiplas. Primeiramente, testa-se a normalidade dos resíduos do espaço amostral com o teste de Shapiro-Wilk [24]. Caso a normalidade seja atendida, então a esfericidade dos dados é verificada com o teste de Mauchly. Em seguida, se a esfericidade é atendida, então o teste de Anova de medidas repetidas é aplicado, caso contrário é feita a correção das amostras por GreenhouseGeisser $(\varepsilon \leq 0,75)$ ou Huynh-Feldt $(\varepsilon>0,75)$ e posteriormente aplicado o teste Anova de medidas repetidas. Caso a normalidade não seja satisfeita, uma transformação de amostras pelo método de boxcox é aplicada, caso resulte em normalidade, então é aplicado o processo descrito anteriormente, caso contrário, o teste de Friedmann é utilizado para a detecção de igualdade entre os espaços amostrais. Por fim, se os dados apresentam distribuição normal, o teste $\mathrm{T}$ pareado com ajuste de Bonferroni para comparações múltiplas é aplicado para detectar a diferença entre as distribuições. Por outro lado, se os dados não apresentam distribuição normal então o teste de Dunn com ajuste de Bonferroni é aplicado [25]. O nível de significância $(\alpha)$ para os testes estatísticos foi de $5 \%$.

\section{Resultados}

Resultados são apresentados para três SNRs: $-3 \mathrm{~dB}$ (SNR muito baixa), $3 \mathrm{~dB}$ (SNR baixa) e $9 \mathrm{~dB}$ (SNR média).

Os resultados para $\mathrm{SNR}=-3 \mathrm{~dB}$ são apresentados na Fig. 2. O teste de Friedman apontou que há pelo menos uma distribuição que é diferente das demais com $p \leq \alpha$. Posteriormente, o teste de Dunn com ajuste de Bonferroni foi aplicado e $p \leq \alpha$ foi alcançado em todos os casos. Assim, podese concluir que a rede neural possui melhor desempenho que a máscara $\mathrm{C} 2 \mathrm{~F}$ utilizando o método de decisão direta para estimativa da $\mathrm{SNR}$. Os resultados para $\mathrm{SNR}=3 \mathrm{~dB}$ são apresentados na Fig. 3, sendo que as mesmas considerações estatísticas obtidas para o caso anterior também foram encontradas. Assim, as mesmas conclusões são válidas.

Por fim, a Fig. 4 apresenta os resultados para a SNR de 9 dB. Nota-se que as distribuições se assemelham e uma inteligibilidade de $90 \%$ é alcançada para todas elas. O teste de Friedmann apontou que há pelo menos uma distribuição diferente das demais, assim, com o teste de Dunn e ajuste de Bonferroni, conclui-se que a distribuição da máscara estimada pela rede neural e da máscara ideal são iguais com $p>\alpha$. O resultado é plausível, visto que há predominância da fala e os métodos geralmente apresentam alta inteligibilidade nesses casos.

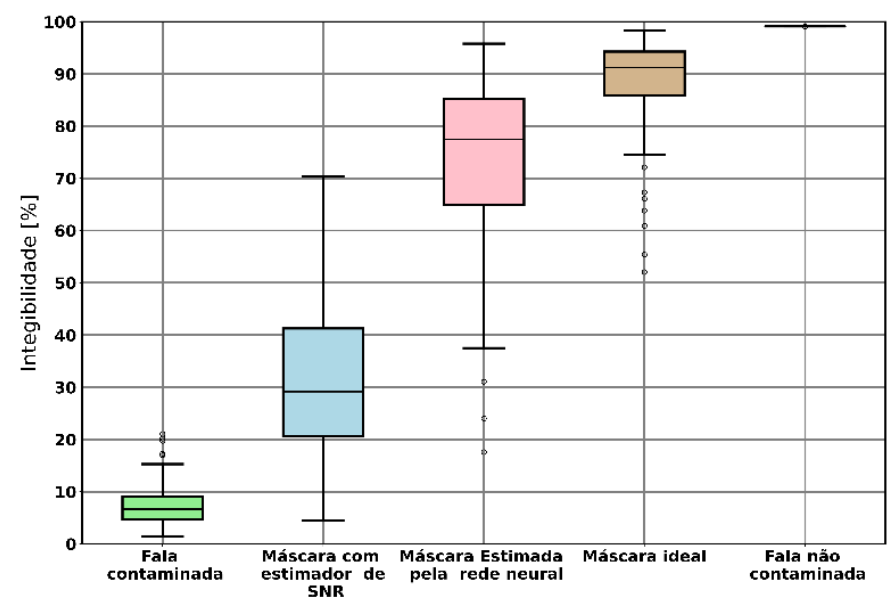

Fig. 2: Inteligibilidade para uma $\mathrm{SNR}=-3 \mathrm{~dB}$.

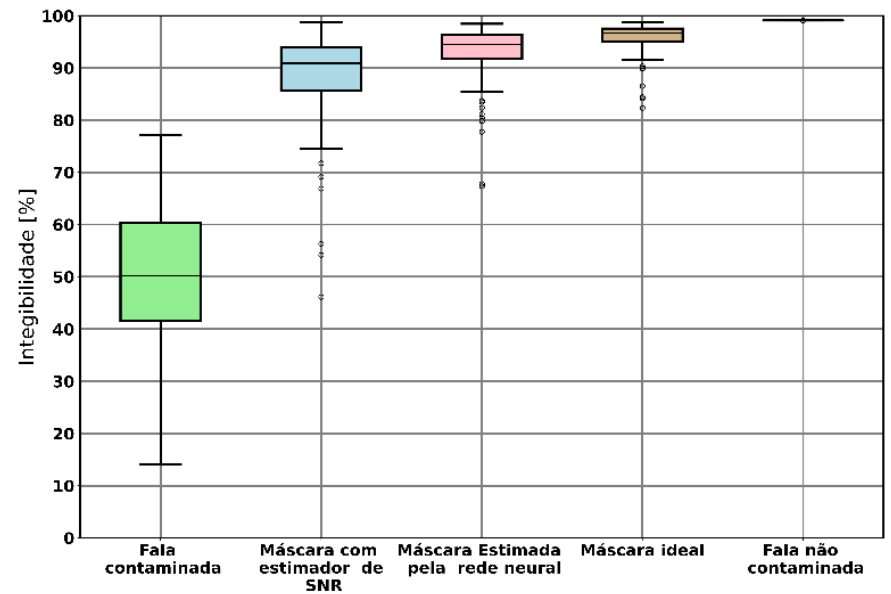

Fig. 3: Inteligibilidade para $\mathrm{SNR}=3 \mathrm{~dB}$.

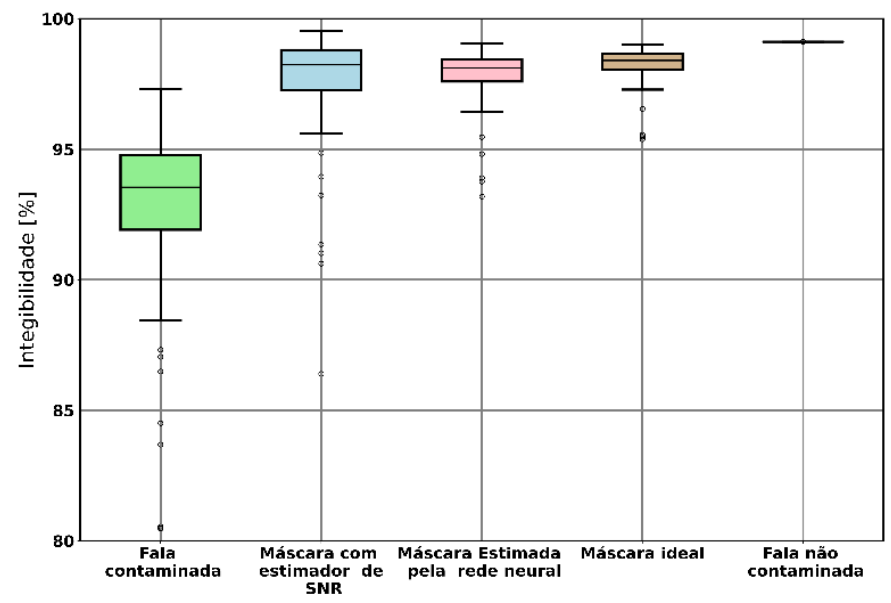

Fig. 4: Inteligibilidade para $\mathrm{SNR}=9 \mathrm{~dB}$.

\section{CONCLUSÕES}

Este trabalho apresentou um método de redução de ruído para implantes cocleares baseado em redes neurais recorrentes. Para treinamento é utilizada uma máscara tempo-frequência para preservação da envoltória da fala e SNR ideal. Simulações computacionais demonstram o melhor desempenho, em termos de inteligibilidade, da estrutura proposta em comparação à máscara de preservação de envoltória utilizando o método de decisão direta para estimação da SNR. Os resultados 
apresentados corroboram a robustez e eficácia de métodos de aprendizado de máquina em comparação aos métodos convencionais, ao custo de um elevado aumento na complexidade computacional.

\section{REFERENCIAS}

[1] J. Wouters, H. J. McDermott e T. Francart, "Sound coding in cochlear implants", IEEE Signal Processing Magazine, v. 32, pp. 67-80, 2015.

[2] C.T. Choi e Y. Lee. "A review of stimulating strategies for cochlear implants", Cochlear Implant Research Updates, v. 16, pp. 77-90, 2012.

[3] A. Hast, et al., "Speech perception of elderly cochlear implant users under different noise conditions", Otology and Neurotology, v. 36, pp. 16381643,2015

[4] R. Koning, et al., "Perceptual and model-based evaluation of ideal timefrequency noise reduction in hearing-impaired listeners", IEEE Transactions on Neural Systems and Rehabilitation Engineering, v. 26, pp. 687-697, 2018.

[5] R. A. Chiea, M. H. Costa e G. Barrault, "New insights on the optimality of parameterized Wiener filters for speech enhancement applications", Speech Communication, v. 109, pp. 46-54, 2019.

[6] R. A. Chiea, M. H. Costa e G. Barrault, "Uma comparação entre máscaras tempo-frequência para redução de ruído em implantes cocleares", Simpósio Brasileiro de Telecomunicações e Processamento de Sinais, pp. 1-5, 2019.

[7] R. Koning, N. Madhu e J. Wouters, "Ideal time-frequency masking algorithms lead to different speech intelligibility and quality in normalhearing and cochlear implant listeners", IEEE Transactions on Biomedical Engineering, v. 62, pp. 331-341, 2014.

[8] R. A. Chiea, M. H. Costa e J. Cordioli, “An optimal envelope-based noise reduction method for cochlear implants: an upper bound performance investigation", IEEE/ACM Transactions on Audio, Speech, and Language Processing, v. 29, pp. 1729-1739, 2021.

[9] R. A. Chiea e M. H. Costa, "Análise de robustez de um método de redução de ruído para implantes cocleares", Simpósio Brasileiro de Telecomunicações e Processamento de Sinais, pp. 1-5, 2020.

[10] T. Goehring, et al., "Speech enhancement based on neural networks improves speech intelligibility in noise for cochlear implant users", Hearing Research, v. 344, pp. 183-194, 2017.

[11] S. W. Fu et al., "End-to-end waveform utterance enhancement for direct evaluation metrics optimization by fully convolutional neural networks", IEEE/ACM Transactions on Audio, Speech, and Language Processing, v. 26, pp. 1570-1584, 2018.

[12] Y. Lai, et al., "A deep denoising autoencoder approach to improving the intelligibility of vocoded speech in cochlear implant simulation", IEEE Transactions on Biomedical Engineering, v. 64, pp. 1568-1578, 2017.

[13] T. Goehring, et al., "Using recurrent neural networks to improve the perception of speech in non-stationary noise by people with cochlear implants", The Journal of the Acoustical Society of America, v.146, pp. 705-718, 2019.

[14] J. Justice, "Analytic signal processing in music computation", IEEE Transactions on Acoustics, Speech, and Signal Processing, v. 27, pp. 670684, 1979.

[15] P. C. Loizou, Speech Enhancement - Theory and Practice $2^{\text {nd }}$, CRC Press, 2013.

[16] D. Ditter e T. Gerkmann, "A multi-phase gammatone filterbank for speech separation via tasnet", IEEE International Conference on Acoustics, Speech and Signal Processing, pp. 36-40, 2020.

[17] IEEE. "Recommended practice for speech quality measurements," IEEE Transactions on Audio and Electroacoustics, v. 17, pp. 225-246, 1969.

[18] Y. Wu, et al., "Characteristics of real-world signal to noise ratios and speech listening situations of older adults with mild to moderate hearing loss", Ear and Hearing, v. 39, pp. 293-304, 2018.

[19] N. Srivastava et al., "Dropout: a simple way to prevent neural networks from overfitting", The Journal of Machine Learning Research, v. 15, pp. 1929-1958, 2014.

[20] J. Xie e M. Zhu, "Investigation of acoustic and visual features for acoustic scene classification”, Expert Systems with Applications, v. 126, pp. 20-29, 2019.
[21] G. L. Mourão, M. H. Costa e S. Paul, "Speech intelligibility for cochlear implant users with the MMSE noise-reduction time-frequency mask", Biomedical Signal Processing and Control, v. 60, pp. 101982-11, 2020.

[22] X. Li, et al., "Non-stationary noise power spectral density estimation based on regional statistics", IEEE International Conference on Acoustics, Speech and Signal Processing, pp. 181-185, 2016.

[23] J. F. Santos, et al., "Objective speech intelligibility measurement for cochlear implant users in complex listening environments", Speech Communication, v. 55, pp. 815-824, 2013.

[24] P. H. Gubert, et al., "The performance impact of data augmentation in CSPbased motor-imagery systems for BCI applications", Biomedical Signal Processing and Control, v. 62, pp. 102152-12, 2020.

[25] A. C. Elliot e W. A. Woodward, Statistical Analysis Quick Reference Guidebook: With SPSS examples, Sage, 2007. 